\title{
De la bière de banane au soda en bouteille. Religion et boisson chez les Rwa du Mont Meru Tanzanie du Nord
}

In: Journal des africanistes. 2001, tome 71 fascicule 2. pp. 77-94.

\begin{abstract}
Résumé
Le passage de la bière de banane au soda en bouteille et au thé, chez les Rwa de Tanzanie du Nord, n'est pas un simple changement de boisson. C'est une véritable mutation sociale, qui les a porté en un siècle d'une société où la bière occupait une place essentielle dans les échanges et la convivialité, vers une société moderne axée sur l'économie de marché. Dans cette mutation, le luthéranisme a joué un rôle de premier plan, en sapant les valeurs morales et les formes de sociabilité qui prévalaient auparavant et en favorisant incidemment l'introduction de la caféiculture. Mais l'accroissement démographique a été tel que les terres riches et fertiles du Mont Meru, où se pratique aujourd'hui une agriculture très intensive, ne suffisent plus à nourrir sa population. Ceci pose une kyrielle de problèmes économiques pour les familles, à la recherche d'ajustements de toutes sortes.
\end{abstract}

\section{Abstract}

Within a century, the Rwa of Mount Mera in Northern Tanzania switched from consuming banana beer to drinking tea and bottled sodas. This however is not a mere change of beverages, it was a sign of deeper social changes. Beer drinking, which was a priviledge of the elderly, was strongly condemned by the Lutheran church. Incidentally coffee farming spread outside the missions and Rwa farmers were incorporated further into the market economy. As a result, new social models developed. Nowadays, the population increase on Mount Meru leads to land shortage, which creates a series of economic and social problems.

Citer ce document / Cite this document :

Baroin Catherine. De la bière de banane au soda en bouteille. Religion et boisson chez les Rwa du Mont Meru Tanzanie du Nord. In: Journal des africanistes. 2001, tome 71 fascicule 2. pp. 77-94.

doi : $10.3406 /$ jafr.2001.1270

http://www.persee.fr/web/revues/home/prescript/article/jafr_0399-0346_2001_num_71_2_1270 
Catherine BAROIN*

\section{De la bière de banane au soda en bouteille* Religion et boisson chez les Rwa du Mont Meru (Tanzanie du Nord)}

\section{Résumé}

Le passage de la bière de banane au soda en bouteille et au thé, chez les Rwa de Tanzanie du Nord, n'est pas un simple changement de boisson. C'est une véritable mutation sociale, qui les a porté en un siècle d'une société où la bière occupait une place essentielle dans les échanges et la convivialité, vers une société moderne axée sur l'économie de marché. Dans cette mutation, le luthéranisme a joué un rôle de premier plan, en sapant les valeurs morales et les formes de sociabilité qui prévalaient auparavant et en favorisant incidemment l'introduction de la caféiculture. Mais l'accroissement démographique a été tel que les terres riches et fertiles du Mont Meru, où se pratique aujourd'hui une agriculture très intensive, ne suffisent plus à nourrir sa population. Ceci pose une kyrielle de problèmes économiques pour les familles, à la recherche d'ajustements de toutes sortes.

Mots clés

Tanzanie, Rwa, bière, religion, café

\section{Abstract}

Within a century, the Rwa of Mount Meru in Northern Tanzania switched from consuming banana beer to drinking tea and bottled sodas. This however is not a mere change of beverages, it was a sign of deeper social changes. Beer drinking, which was a priviledge of the elderly, was strongly condemned by the Lutheran church. Incidentally coffee farming spread outside the missions and Rwa farmers were incorporated further into the market economy. As a result, new social models developed. Nowadays, the population increase on Mount Meru leads to land shortage, which creates a series of economic and social problems.

\section{Keywords}

Tanzania, Rwa, beer, religion, coffee

\footnotetext{
* CNRS, UMR 7041, Nanterre, baroin@mae.u-paris10.fr

** Cet article a fait l'objet d'une présentation au XVII ${ }^{\circ}$ colloque de la Commission internationale d'anthropologie de l'alimentation (ICAF) et de l'Union internationale des sciences anthropologiques et ethnologiques (IUAES), à Borja (Espagne), les 22-24.11.01.
} 
La bière, de mil ou de banane, joue dans nombre de sociétés africaines un rôle social de premier plan. Elle est source de convivialité, moteur de la production économique, moyen d'échange aussi bien entre les vivants qu'avec les morts. D'un bout du continent à l'autre, voire au-delà, des pratiques parfois très proches s'observent en dépit des distances culturelles et géographiques, à tel point qu'une anthropologie comparée des sociétés de buveurs de bière produirait d'intéressants résultats. J'y apporterai ici une modeste contribution, en traitant du rôle social de la bière chez les Rwa, et de son déclin par suite des bouleversements sociaux qu'ils ont connus au cours du siècle écoulé.

\section{L'ETHNIE RWA}

Les Rwa, connus localement sous le nom de Meru', sont établis sur les flancs sud-est du Mont Meru (4585 m), face au Kilimandjaro. Ils sont plus de 150000 à l'heure actuelle, dans un environnement naturel très favorable car les sols de ce volcan sont riches et bien arrosés. Comme leurs voisins les Arusha, nilotes établis à l'ouest sur la même montagne, et comme leurs parents les Chaga du Kilimandjaro, les Rwa associent une variété de cultures où prédominent le café, culture de rente, et la banane, culture vivrière. Ils complètent ces ressources par un élevage de petit bétail ou de vaches en stabulation, et par des cultures de maïs et de haricot dans les plaines plus sèches en contrebas de la montagne. Leur habitat est dense et dispersé.

L'ethnie rwa n'est pas très ancienne. Elle s'est peu à peu constituée au cours du XVII ${ }^{\text {ème }}$ siècle. Le Mont Meru était alors inhabité, si ce n'est par quelques groupes épars de chasseurs-cueilleurs, actuellement disparus. Aussi put-il tenir lieu de refuge à des migrants d'origines diverses, Shambaa des Monts Usambara, Maasai des plaines avoisinantes, ou Chaga du Kilimandjaro voisin, notamment les Chaga Machame. La langue que parlent aujourd'hui les Rwa, le $k i-r w a^{2}$, est une langue bantoue proche du ki-chaga parlé par les Machame, avec lesquels ils partagent de nombreux traits culturels.

Il existe peu de témoignages sur la société rwa précoloniale car, contrairement aux Chaga, les Rwa sont restés à l'écart du commerce caravanier qui drainait escaves et ivoire vers la côte de l'océan Indien. Toujours est-il que les Rwa aujourd'hui conservent de leur passé une image,

\footnotetext{
${ }^{1}$ Ils n'ont aucun lien de parenté avec les Meru du Mont Kenya.

${ }^{2}$ Le ki-rwa est une langue à tons, mais la transcription adoptée ici est simplifiée. Les tons ne sont pas transcrits. La majuscule $R$ correspond au $r$ labialisé, tandis que le groupe $s h$ se prononce comme en anglais.
} 
une image idyllique. Leurs ancêtres, selon eux, menaient une vie de cocagne, où la bière coulait à flots et la viande était abondante. Il suffisait à un homme, pour s'enrichir, d'épouser de nombreuses femmes afin de cultiver des surfaces plus grandes et s'assurer une descendance plus nombreuse.

Sans doute est-il naturel d'embellir, à mesure que l'on vieillit, le souvenir de sa jeunesse. Cependant les premiers observateurs occidentaux, à la fin du XIX ${ }^{\text {eme }}$ siècle et au début du $\mathrm{XX}^{\text {ème }}$, confirment ce tableau. Les hommes rwa, selon eux, coulaient des heures oisives, à se reposer ou à boire de la bière, en grande quantité ${ }^{3}$, tandis que les femmes travaillaient aux champs. De grands festins de viande avaient lieu de temps à autre, où un homme riche pouvait se permettre d'étrangler par pendaison plusieurs bœufs en une seule occasion afin de régaler, de viande et de bière, ses amis et connaissances ${ }^{4}$. La polygamie était très répandue. Les hommes les plus riches avaient plus de 10 femmes et, encore de nos jours, avoir 3 ou 4 épouses n'a rien d'inhabituel pour les hommes les plus âgés.

La nostalgie que gardent les anciens de cette période d'abondance est d'autant plus forte que, maintenant, le sentiment de pénurie domine. Beaucoup de pères de famille s'inquiètent de ne pouvoir procurer, à chacun de leurs fils, un lopin de terre suffisant pour vivre. Par contraste, ils regrettent ce passé où les terres étaient plus vastes qu'on n'en pouvait cultiver, et ils oublient que la vie, en fait, n'était pas rose pour tout le monde dans ce passé un peu mythique. L'insécurité était forte, le statut des femmes et des jeunes très médiocre, et le niveau de vie dans l'ensemble assez bas, alors qu'il s'est accru par la suite avec l'argent du café. Mais la discipline régnait sans partage, alors que certains voyous osent aujourd'hui contester leurs aînés. Beaucoup de familles, certes, profitent de biens de consommation modernes, mais des aspirations nouvelles se sont développées simultanément, sources de frustrations.

La société rwa, en un siècle, a donc profondément changé. Elle a basculé d'une logique d'échange et de convivialité, d'une économie d'abondance où les richesses étaient consommées en commun (du moins par les hommes d'âge mûr détenteurs de l'autorité), à une logique plus individualiste où la consommation de bière perd du terrain en même temps que l'autorité des anciens.

La bière, comme autrefois, se fabrique avec des bananes et du malt d'éleusine. Elle se dénomme waRi, que l'on précise à l'occasion en waRi wa umbi, "bière d'éleusine", pour l'opposer à l'hydromel ou "bière de miel" waRi wa ukyi. Les bananes mûres sont pelées, coupées en morceaux, et

${ }^{3}$ D'après un missionnaire allemand, en 1903, "One practically never finds (the men) at work, but usually lying around lazily or sitting and drinking beer" (Spear 1995:28).

${ }^{4}$ Baltazar Kaaya, 1978. 
mises à bouillir de longues heures dans de l'eau, jusqu'à ce que la couleur devienne rouge. Le mélange est alors versé dans de vastes récipients, et laissé trois jours à découvert. Puis on ajoute de l'eau pour un jour de plus. Ensuite cette bouillie est filtrée. Aujourd'hui, c'est une toile de jute qui remplace l'ancien filtre confectionné avec des fougères ou avec les feuilles de l'arbre $n R u k a^{5}$.

Parallèlement, les grains d'éleusine, cultivée essentiellement à cette fin, sont placés sur des feuilles de bananiers, mouillés et recouverts d'autres feuilles de bananiers. Ainsi protégés, ils germent au bout de quelques jours. On les découvre alors et les laisse sécher à l'air libre, ce qui prend quelques jours de plus. Puis on les broie, et le produit obtenu est versé dans une grande marmite où il est cuit à l'eau comme du porridge. Ensuite, le jus extrait de la bouillie de banane est ajouté à ce porridge d'éleusine, avec de l'eau. Ce mélange préparé le soir est laissé à reposer pendant la nuit, et la bière est prête le lendemain.

Cette boisson, qui jouait jadis un rôle si central chez les Rwa, nous servira donc de fil directeur pour retracer l'évolution de leur société. Dans une première partie, nous dresserons un état des lieux de la société rwa au tout début de la période coloniale, c'est-à-dire vers la fin du XIX ${ }^{\text {ème }}$ et le début du $\mathrm{XX}^{\text {ème }}$ siècle, en y soulignant l'importance matérielle, sociale et rituelle de la bière. Puis, dans une seconde partie, nous montrerons comment l'évolution des manières de boire traduit la cascade de changements déclenchés par le développement concomitant du luthéranisme et de la culture du café.

\section{LA SOCIETE RWA AU DEBUT DU XX ${ }^{\text {EME }}$ SIECLE}

Il y a cent ans environ, la société rwa était organisée selon deux axes complémentaires. Un système d'âge et de génération, qui répartissait les hommes en classes de circoncision successives, établissait des solidarités extra-familiales entre hommes d'une même classe, chargés d'assurer le maintien de l'ordre et le respect des aînés. Ce système était recoupé par une organisation en clans patrilinéaires, qui géraient les questions familiales et foncières. En outre, la société rwa avait à sa tête un "chef" mangi choisi dans un clan dominant, celui des Kaaya, tandis qu'un autre clan, celui des Mbise, avait la préséance en matière religieuse pour organiser les rituels de pluie.

\footnotetext{
5 Non identifié.
} 


\section{Les chefs}

Les prérogatives des "chefs", avant la colonisation, ne sont pas claires (Spear 1997:27). Il semble qu'ils n'aient jamais eu beaucoup d'autorité. La tradition conserve une liste de seize "chefs", neuf depuis les origines jusqu'à la fin du XIX ${ }^{\text {ème }}$ siècle, suivis de sept autres jusqu'à l'indépendance du Tanganyika et l'abolition des chefferies traditionnelles. Mais la colonisation transforma ces chefs en agents de sa politique, chargés de percevoir l'impôt.

\section{Le système d'âge}

Le système d'âge et de génération des Rwa rappelle celui d'autres sociétés d'Afrique de l'Est ${ }^{6}$. Des "classes d'âge" irika (pl. marika), regroupant chacune des hommes d'âge voisin, se succèdent depuis les origines, dont la liste s'allonge au fil du temps. Elle comptait seize noms au début du $\mathrm{XX}^{\text {ème }}$ siècle, et en comporte six de plus aujourd'hui.

La dernière classe est celle des "guerriers" nsero (pl. vasero), dont le statut s'oppose à celui de tous leurs "aînés" nsuri (pl. vasuri). Ils assurent la défense du pays sous l'autorité de leurs "pères", qui appartiennent à la deuxième classe au-dessus d'eux. Dans le même temps, une nouvelle classe en formation incorpore peu à peu tous les jeunes hommes, à mesure qu'ils sont initiés. L'initiation se fait individuellement ou par petits groupes. Elle comporte la circoncision, suivie d'une période de réclusion où les circoncis reçoivent un enseignement tenu secret pour les femmes et les enfants. Ces nouveaux initiés sont les "fils" de la classe immédiatement antérieure à celle des guerriers, car il y a toujours un écart d'au moins deux classes entre un père et son fils.

Au bout de quinze à vingt ans, les guerriers n'ont plus l'âge d'assurer la défense du pays mais surtout, leurs propres fils réclament d'être circoncis à leur tour. Il faut, pour cela, créer une nouvelle classe car les fils ne peuvent appartenir à la classe qui suit celle de leurs pères. Comme le recrutement ne peut se faire que dans une classe à la fois, on clôture donc la précédente, et tout le système se décale d'un cran. Les guerriers deviennent des "anciens" après avoir transféré leur rôle à la classe qui les suit.

Ce basculement est ponctué par une très grande fête, où les guerriers qui se retirent transfèrent solennellement leur statut à la classe suivante. Des ripailles somptueuses sont arrosées d'énormes quantités de bière, aux frais des futurs retraités. Plus tard, une autre fête marquera le moment où les "pères" choisiront un nom pour la nouvelle classe, celle de leurs "fils", où l'on boira beaucoup de bière également.

\footnotetext{
${ }^{6}$ Il est voisin de celui des Meru Tigania-Igembe du Kenya étudiés par A.-M. Peatrik (1995, 1999).
} 
Ce système n'est pas seulement source de grandes festivités. Il organise la vie des hommes en stades successifs (non-initié, initié, guerrier, ancien), permet aux anciens de contrôler les jeunes, suscite de fortes solidarités au sein de chaque classe, et garantit une défense plus efficace du pays, grâce à l'organisation des guerriers.

La bière y joue un rôle essentiel. Elle n'est pas seulement bue à profusion lors des fêtes, elle est aussi un signe de statut, car seuls les aînés sont (étaient) autorisés à en boire. Elle aurait pu, chez les Rwa, donner lieu à un adage : "Dis-moi ce que tu bois, et je te dirai qui tu es". Tandis que les guerriers assuraient la sécurité et la réalisation des grands travaux décidés par les anciens, l'occupation essentielle de ces derniers consistait à boire de la bière et à discuter ensemble des affaires du pays.

De plus, la bière était parfois le moyen de communiquer un message. Ainsi les fils des guerriers, pour réclamer leur initiation (et donc le basculement du système), ne s'exprimaient pas directement en ces termes. Ils sollicitaient "l'autorisation de faire de la bière" en s'adressant aux deux hommes les plus jeunes de la catégorie de leurs "pères". La bière tenait donc lieu de langage symbolique dans la formulation leur demande. Et lorsqu'à titre exceptionnel, un père désirait voir son fils intégrer la génération précédant celle qu'il aurait dû intégrer, c'est en offrant de la bière et de la viande aux membres de cette génération qu'il effectuait sa demande, ce qui ne lui en garantissait d'ailleurs nullement l'acceptation. Ce rôle symbolique de la bière comme sa consommation abondante, lors des réunions masculines et des cérémonies, se retrouvent dans la vie des clans.

\section{Les clans}

Les clans patrilinéaires, au nombre d'une quinzaine, étaient assez autonomes. Chacun avait un chef nshili wa ufwaRi, formait une unité géographique, et se caractérisait par son ou ses lieux de culte distincts, ainsi que sa tradition liée à l'histoire de l'ancêtre fondateur.

$\mathrm{Au}$ sein du clan se réglaient toutes les questions relevant de la vie privée, c'est-à-dire avant tout les affaires familiales et foncières. Par exemple les dissensions conjugales ou familiales, les contestations en matière d'héritage, le sort de la veuve et des orphelins, les problèmes de limites de champs étaient d'abord soumis au chef de la famille étendue (composée du père et de ses fils mariés, établis près de lui). A défaut de solution, il était fait appel à l'arbitrage des hommes du lignage, et s'il s'avérait insuffisant, c'est l'ensemble du clan qui se réunissait pour discuter et trancher la question.

Une telle réunion ne se concevait pas sans bière à consommer. Toute personne souhaitant soumettre un problème à l'arbitrage du clan devait 
d'abord fournir de la bière, que les membres du clan boiraient tout en débattant, à la recherche d'une solution. La bière était le moteur de toute réunion : indispensable pour que les hommes se rassemblent, et nécessaire pour stimuler les débats.

Le clan n'avait pas seulement autorité en matière juridique, il était aussi (et il est encore) porteur d'une identité collective, source de solidarité matérielle et morale entre ses membres. Le meurtre au sein du clan était une affaire interne qui ne donnait pas lieu à compensation. Par contre si la victime était étrangère au clan, tous ses membres se cotisaient pour payer la compensation réglementaire de 49 bovins au clan du défunt. Sinon, c'est l'ensemble du clan meurtrier qui serait exposé à la vengeance du clan agressé. La tradition prévoyait aussi un barème d'indemnités en cas de coups et blessures, le plus souvent des quantités de bière variant selon la gravité du dommage. Par ailleurs, une mauvaise action cachée ou impunie entraînait de redoutables châtiments surnaturels (stérilité, maladie ou mort) soit pour le coupable ou les siens (enfants, champs et troupeaux), soit pour l'un ou plusieurs des membres de son clan. Tout malheur était interprété en ce sens, et l'exposition à une même menace était bien le signe d'une identité de substance entre membres d'un même clan. Le clan, sorte de prolongement naturel de la famille, était impliqué dans les grands événements familiaux tels que les mariages. Après accord entre deux familles, un projet de mariage s'officialisait par le don d'une grande quantité de bière, de la famille du futur marié au clan de la fiancée. Conviés à partager cette bière, les membres du clan de celle-ci marquaient en la buvant qu'ils prenaient acte du projet d'union et y consentaient ${ }^{7}$. Cette consommation de bière revêt donc un caractère mi-convivial, mi-rituel. Ce deuxième aspect prédomine dans la vie religieuse des Rwa au début du $\mathrm{XX}^{\text {ème }}$ siècle.

\section{La vie rituelle et religieuse}

Avant le christianisme, la religion des Rwa était essentiellement axée sur le culte des ancêtres, qu'il s'agisse de ceux de la famille, du lignage, du clan, ou de la communauté dans son ensemble. Il importait de s'attirer leur bienveillance par des offrandes fréquentes, pour se garantir bien-être et prospérité. A l'inverse, lorsqu'une difficulté survenait (sécheresse, stérilité, accident, maladie ou mort), on soupçonnait tout de suite qu'un ancêtre puisse en être la cause, et se venge ainsi d'avoir été insuffisamment honoré. C'est donc par une offrande réparatrice à l'ancêtre mécontent qu'on espérait remédier au problème. Trois types de denrées étaient offertes aux ancêtres :

\footnotetext{
${ }^{7}$ Il en est de même chez les Dogon (Jolly 1995:439)
} 
de la bière (pour les ancêtres masculins), du lait (pour les ancêtres féminins), et des animaux, sur pied ou sacrifiés pour la circonstance.

Le plus grand rituel, chaque année, était un rituel de pluie, qui eut lieu encore en 1984 et 1989. Son souvenir reste très vivant dans les mémoires. Une procession menée par des membres du clan Mbise, au nom de l'ensemble des Rwa, montait vers la caverne de l'ancêtre fondateur, Lamirei, située dans le cratère du Mont Meru. On lui portait des offrandes, d'hydromel pour lui, de lait pour sa femme, plus deux moutons noirs qu'on laissait sur place, et l'on priait Lamirei d'accorder la pluie. L'hydromel offert lors de ce rituel reste aujourd'hui la boisson préférée des hommes âgés, mais la bière de banane le remplace depuis longtemps dans la plupart des usages, y compris religieux.

D'autres rituels, collectifs ou individuels, ponctuaient il y a un siècle la vie quotidienne. Chaque clan possédait son lieu de culte, un grand arbre ou une colline par exemple, et y faisait régulièrement des offrandes à ses ancêtres. Chaque chef de famille honorait ses ascendants directs en particulier lors des principaux événements familiaux tels que naissance, imposition du nom, ou funérailles. Par une libation de bière ou de lait sur l'autel familial (le plus souvent un rocher ou un arbre) il associait les ancêtres aux événements et se conciliait leur bienveillance. Du sang ou des morceaux d'un animal sacrifié s'ajoutaient plus rarement aux offrandes, en particulier lors d'une naissance, le reste étant consommé par les vivants. La vie religieuse était donc intimement mêlée à la vie familiale, par une foule de menus gestes, parmi lesquels la bière offerte et consommée tenait une place de premier plan.

\section{La famille}

Mais ce n'est pas seulement avec les morts que la bière permettait d'établir un lien. Elle était aussi le moyen de créer des relations sociales entre les vivants, aussi bien d'une manière concrète par le partage, que d'une manière symbolique par l'offrande. Tel était le cas aussi bien dans le système d'âge et l'organisation clanique, que dans le cadre familial.

L'importance symbolique de la bière se marque dans les conceptions relatives à la gestation de l'être humain. La grossesse était assimilée au processus de fabrication de la bière, comme le dénote ce proverbe : "Du grain trop mûr ne donne pas de bonne bière" Mimia shisha iraa waRi ndi, qui signifie qu'une femme trop âgée ne peut produire un bel enfant (Harjula 1997:101). La bonne bière y est une image pour un bébé en bonne santé8.

\footnotetext{
${ }^{8}$ Chez les Dogon aussi, qui sont de grands buveurs de bière comme les Rwa et chez lesquels la bière occupe un rôle social comparable, un parallèle est établi entre le brassage de la bière
} 
Chez les Rwa du début du $\mathrm{XX}^{\text {ème }}$ siècle, il était exclu pour une jeune femme d'enfanter en dehors du mariage. Elle était d'abord soumise à un long processus, conduisant de la demande en mariage jusqu'à l'installation définitive du couple. D'une famille à l'autre, cela impliquait une série de dons, et en particulier des dons de bière. Ainsi, pour formuler une demande en mariage, il était nécessaire que le père du garçon apporte un demi-seau de bière lors de sa visite au père de la jeune fille. Ensuite, quand la demande en mariage était acceptée, la famille du fiancé devait donner à celle de la future épouse de la bière ou un seau de miel pour fabriquer l'hydromel que boiraient les membres du clan de la fiancée, en signe d'acceptation du projet de mariage. Par ailleurs, les fiançailles étaient également officialisées du côté du jeune homme, par la fabrication d'une importante quantité de bière offerte aux membres de son propre clan.

Divers dons d'animaux faisaient suite, offerts par la famille du garçon à certains parents et parentes de la future mariée, suivis d'un nouveau don de bière au père de la jeune fille, pour le prier de fixer la date du mariage. Il était nécessaire, pour que la cérémonie ait lieu, que le marié soit circoncis, et la mariée excisée. Cette dernière l'était en présence de son futur époux, puis elle restait recluse jusqu'à cicatrisation (Puritt 1970:81). Par la suite, le marié demandait à son beau-père l'autorisation d'emmener sa femme chez lui, et lui faisait un nouveau don de bière à cette fin. Au total la tradition prévoyait donc toute une série de dons, marquant les étapes successives du processus matrimonial, parmi lesquels la bière, offerte en quantités précises, à des fins et à des moments précis, tenait une place importante.

Les femmes mariées, comme aujourd'hui, quittaient leur famille pour vivre chez leur mari, chaque homme marié recevant alors de son père les champs nécessaires à sa subsistance. L'autorité du père sur ses fils, même après leur mariage, restait considérable et ils vivaient proches les uns des autres. Le plus jeune fils avait la charge de ses parents devenus vieux, et recevait la plus grosse part d'héritage. Cette situation, comparable à celle qui s'observe chez les Chaga du Kilimandjaro, ne manquait pas de susciter de fortes jalousies entre frères, comme le souligne Moore (1986) à propos des Chaga.

En cas d'inconduite, un père pouvait maudire son fils, et les conséquences d'un tel geste étaient très redoutées. La malédiction toutefois pouvait être lavée si le fils faisait amende honorable. Il devait pour cela tuer un bouc et offrir de la bière à son père. Les membres de la classe d'âge du

et la gestation d'un enfant (Jolly 1995:490). L'analogie qu'expriment ainsi ces grands buveurs, qui n'ont ni parenté ni proximité géographique, n'a sans doute rien d'unique. 
père intervenaient alors : ils crachaient dans la bière que devait boire le fils, ce qui annulait la malédiction.

Si la bière permettait d'annuler une malédiction, à l'inverse elle était aussi le véhicule privilégié de l'empoisonnement par les "sorciers" nsavi (pl. vasavi). C'est pourquoi celui qui offre de la bière la goûte avant de la proposer à son hôte, pour le rassurer sur l'innocuité du breuvage. Ceci s'appelle "retirer le cafard" uta ngishe tafo'. Dans le même ordre d'idée, l'épouse goûtait devant son mari la nourriture préparée pour lui, habitude que les couples âgés conservent de nos jours.

La polygamie était pratiquée à vaste échelle, car la terre était abondante. Chaque co-épouse cultivait ses champs et gérait indépendamment sa maisonnée, mais la terre, comme le bétail, appartenait aux hommes.

\section{L'économie}

Basée sur l'agriculture et l'élevage, l'économie des Rwa bénéficiait de conditions écologiques particulièrement favorables. Les riches sols volcaniques du Mont Meru profitent de deux saisons des pluies annuelles, ainsi que d'une irrigation régulière le reste de l'année, à partir des torrents descendant de la montagne. La fertilité des champs était renforcée par un apport régulier de fumier, grâce à l'élevage de bovins et de petit bétail. Les bananeraies, qui demandent peu de travail et dont la production s'échelonne sur l'année entière, assuraient une alimentation régulière.

Les troupeaux, vaches et petit bétail, pâturaient sur des prés collectifs sous la surveillance des enfants. Ils servaient aux échanges, notamment pour le mariage, et permettaient aux hommes d'organiser de grands festins de viande. Les bananes et le maiis formaient la base de la nourriture, complétée de viande ou de haricots, ou encore d'autres légumes.

La bière, fabriquée en quantités colossales, était la boisson sociale par excellence. Elle avait sa place à toutes les étapes de l'existence, de la naissance à la mort et même au-delà, puisque les morts aussi recevaient leur part de bière. Elle accompagnait toutes les réunions masculines importantes. Elle marquait l'opposition statutaire entre les "guerriers" vasero et les "hommes d'âge mûr" vasuri. Pour ces derniers, boire de la bière constituait en fait la principale occupation ${ }^{10}$. Bref, cette boisson était fabriquée, donnée, reçue et consommée en abondance dans une société d'abondance, où les rôles étaient bien définis : les femmes assuraient la production de nourriture, les guerriers la défense du pays, et les hommes d'âge mûr pouvaient rester

\footnotetext{
9 Je n'ai pu obtenir aucune information sur le symbolisme du cafard, en cause dans cette expression.

${ }^{10}$ Cf. Spear (1995:28) et Puritt (1970:103).
} 
oisifs, discuter ensemble et boire de la bière à satiété. Cependant l'insécurité était grande, et la fin du XIX ${ }^{\text {ème }}$ siècle fut marquée par un cumul de catastrophes écologiques qui précédèrent de peu la colonisation, celle-ci entraînant à son tour une cascade de bouleversements plus profonds encore.

\section{UN SIECLE DE BOULEVERSEMENTS SOCIAUX}

Je ne ferai ici qu'une brève esquisse des changements qui, en un siècle, affectèrent de fil en aiguille tous les aspects de la société rwa. L'importance sociale de la bière, soulignée dans la partie qui précède, restera le fil directeur de cette description, car les modes de consommation de la bière se modifièrent parallèlement peu à peu, ce qui ne fait que traduire la profondeur d'autres mutations.

\section{Les premiers contacts avec les Blancs}

A la fin du XIX ${ }^{\text {ème }}$ siècle, une série de cataclysmes (peste bovine, variole, invasions de criquets, sécheresses) affectèrent l'ensemble de l'Afrique de l'Est ${ }^{11}$. La société rwa, bien qu'elle fut peu impliquée dans les échanges commerciaux de l'époque, en fut également affectée, et les angoisses suscitées par ces catastrophes expliquent sans doute l'issue tragique de la première tentative d'implantation des Blancs en pays rwa, en 1896. Il s'agissait de deux missionnaires allemands qui, en dépit de l'accord conclu avec le chef, furent tués par les guerriers rwa. Cet incident témoignait de la faible autorité du chef et marquait d'emblée les Rwa, aux yeux du colonisateur, comme une ethnie rétive.

La répression des Allemands, qui étaient installés depuis peu dans la ville voisine d'Arusha, fut terrible. Le pays rwa fut dévasté. Le chef fut pendu, ses deux successeurs mis en prison, et la chefferie traditionnelle devint rapidement, selon un schéma classique, l'organe d'application de la politique coloniale.

L'échec de 1896 ne découragea pas longtemps la mission de Leipzig. Elle revint s'installer en 1902 avec la protection des Allemands et, pendant plusieurs décennies, l'Eglise luthérienne fut la seule Eglise chrétienne implantée en pays rwa. Mais en dépit du zèle des pasteurs successifs, le christianisme avait peu de succès car la rigidité luthérienne se heurtait à des conceptions diamétralement opposées. En effet, ce n'est pas seulement une religion nouvelle que les pasteurs cherchaient à introduire, ils voulurent aussi imposer d'emblée leur propre code moral et combattre des mœurs qu'ils jugeaient condamnables : l'alcoolisme et l'oisiveté des anciens, la

${ }^{11} C f$. Iliffe 1979:124sqq. 
polygamie, les danses lascives des guerriers et de leurs compagnes, la superstition.

Pour mieux asseoir leur influence sur leurs premiers adeptes, les missionnaires avaient ouvert un internat où ces jeunes, coupés de leur milieu familial, étaient logés et nourris ${ }^{12}$. Ils n'y recevaient pas seulement un enseignement religieux, ils apprenaient aussi à lire, écrire et compter, et travaillaient sur les champs de café qui aidaient à financer la mission. De la sorte l'Eglise ne bornait pas son influence au domaine religieux, elle fut aussi le principal vecteur d'introduction de deux autres facteurs de changement considérables, l'alphabétisation et la culture du café.

\section{Le café}

Sous l'autorité allemande puis anglaise, la pacification du pays avait permit aux Rwa de retrouver assez rapidement leur prospérité, qui prit un nouvel essor avec le développement de la culture du café. Cette dernière fut d'abord introduite chez les paysans rwa par l'intermédiaire des missions. Les élèves, familiarisés avec cette plante sur les champs de la mission, emportèrent chez eux des plants de café. Sous les bananiers, ils commencèrent à cultiver le café à leur profit, en dépit de hostilité des planteurs blancs établis en contrebas, qui souhaitaient conserver le monopole de cette culture très lucrative.

Mais la population rwa s'accroissait et le problème de la pression foncière devint bientôt préoccupant. En effet, toutes les terres cultivables, sur le Mont Meru, avaient été peu à peu défrichées et à sa base, les plantations de café de colons blancs ceinturaient presque entièrement le pays rwa, excluant toute possibilité d'extension géographique et coupant l'accès aux cours d'eau pour abreuver les troupeaux. Pour régler le problème foncier, un expert fut commis, le juge Wilson, qui remit son rapport en 1947. Il y préconisait l'expropriation d'une zone de pâturages à l'est du Mont Meru, pour y créer un nouvel espace réservé aux colons.

Ce projet d'expropriation suscita une résistance obstinée de la part des Rwa. Les premiers chrétiens prirent la tête du mouvement, car ils n'étaient pas seulement planteurs de café, ils savaient aussi lire et écrire. Ils prirent un avocat et intentèrent un procès aux Anglais, qui administraient alors le Tanganyika sous la tutelle des Nations Unies. Cet épisode de lutte anticoloniale, précurseur de l'indépendance, est connu sous le nom de Meru Land Case et reste source d'une grande fierté pour les Rwa. Il fut aussi pour l'Eglise luthérienne l'occasion d'accroître considérablement son influence, et de contribuer ainsi davantage aux bouleversements sociaux déjà amorcés.

${ }^{12} C f$. Spear (1995). 
L'Eglise en effet, à travers ses premiers adeptes qui avaient pris la tête de la résistance aux Anglais et de la coopérative de café ${ }^{13}$, apportait un modèle nouveau de réussite sociale. Forts de leur succès politique, moral et économique, les premiers chrétiens constituaient la nouvelle élite, et leur exemple fut bientôt suivi à vaste échelle par les autres paysans. La conversion au christianisme devint générale et la prospérité apportée par l'argent du café permit aux Rwa, de plus en plus, d'accéder aux biens de consommation nouveaux qu'offrait l'économie de marché.

Bien sûr, cette situation ne pouvait que bouleverser en profondeur les principes d'autorité de la société rwa. Désormais la sagesse et le pouvoir n'étaient plus le monopole des anciens puisque des hommes jeunes, chrétiens et lettrés, ouvraient la voie vers l'indépendance et une prospérité nouvelle.

\section{La bière}

De plus, l'Eglise battait en brèche le prestige des aînés sur le plan moral, en prônant l'abstinence en matière d'alcool. Ce qui était jusque là un privilège, la consommation effrénée de bière par les hommes d'âge mûr, devint une pratique moralement condamnable. L'Eglise donnait ainsi aux jeunes des arguments pour remettre en cause la dignité morale et l'autorité des anciens, ce qui aurait été inconcevable auparavant.

Par ailleurs la consommation de bière ne fut pas seulement réprouvée, elle cessa aussi d'être réservée aux anciens. Certains jeunes, profitant de la liberté financière nouvelle que leur apportait l'économie de marché, en profitèrent pour s'adonner eux-aussi à la boisson, contrevenant ainsi à la fois aux préceptes de l'Eglise et aux règles d'usage anciennes. Parfois même, ils en arrivèrent à insulter leurs aînés sous l'effet de l'ivresse, double manière de leur faire insulte. Le privilège de boire s'était transformé en tare morale et cessait d'être une prérogative liée à l'âge.

\section{$L$ 'autorité des anciens}

Dans son combat spirituel, l'Eglise s'opposa aux pratiques rituelles des Rwa. Avant l'arrivée du christianisme, ceux-ci croyaient déjà en un "Dieu créateur du monde" Iruva. Mais il n'avait, selon eux, pas plus d'influence sur leur existence que leurs ancêtres lointains. Par contre la bienveillance des aînés et des ascendants les plus proches était source de prospérité, et leur malédiction très redoutée. C'était donc eux, et non pas Iruva, qu'il importait d'honorer. Or selon l'Eglise, c'est Dieu lui-même, et lui seul, qui détermine le sort de chacun, et la crainte de la malédiction est impie puisque

${ }^{13}$ Cf. Baroin 1998:542-543. 
c'est une forme de remise en cause de la toute puissance de Dieu. Le bon croyant, selon l'enseignement des luthériens, est celui qui remet son sort exclusivement entre les mains de Dieu, et sa foi le préserve des dangers de la malédiction et de la sorcellerie.

Or le pouvoir de maudire était un attribut essentiel des aînés, dans le cadre de la famille comme dans celui, plus large, du système des classes générationnelles. Sa remise en cause ne pouvait donc que saper davantage l'autorité des aînés sur les jeunes. Ainsi c'est une autorité morale sans partage que les autorités ecclésiastiques cherchaient à gagner aux dépends des anciens.

L'Eglise ne cherchait pas seulement à substituer son autorité à celle des anciens. Elle voyait aussi, dans le système générationnel, un autre ennemi. Au début du christianisme en effet, les nouveaux convertis s'exposaient à la malédiction de la classe de leurs "pères" (ce qui, dans le système rwa, équivalait à une menace de mort) et ils subissaient en outre l'ostracisme de leurs compagnons d'âge. Cette mise à l'écart était une sanction à peine moins redoutable, car la solidarité très vive entre membres d'une même classe était l'un des piliers de la sociabilité rwa. Sur le plan moral, le puritanisme luthérien réprouvait, de plus, les festivités organisées dans le cadre du système d'âge : les beuveries de bière lors des cérémonies générationnelles, et les danses "lascives" des guerriers et de leurs compagnes $^{14}$.

\section{La polygamie}

Un autre aspect important de la vie sociale des Rwa, la polygamie, se trouvait lui aussi condamné, car elle est totalement contraire à la conception du mariage inscrite dans le christianisme. L'attitude de l'Eglise envers le mariage, et plus généralement envers les femmes, contribua d'ailleurs beaucoup à leur conversion en nombre : elles trouvaient dans cette religion nouvelle un meilleur statut moral et social. Les premiers chrétiens durent donc assumer de douloureuses ruptures: non seulement renoncer aux solidarités générationnelles, mais aussi se démarquer sur le plan matrimonial, car un statut de polygame les aurait exclus de l'Eglise.

Sur le plan démographique, l'abandon progressif de la polygamie a peut-être provoqué la croissance, et non le recul de la natalité. En effet dans la société rwa précoloniale, le mari devait interrompre les rapports sexuels avec son épouse après chaque naissance pendant deux ou trois ans, jusqu'au sevrage de l'enfant, et la polygamie lui permettait de satisfaire ses pulsions

\footnotetext{
${ }^{14}$ Qui n'étaient pourtant pas associées à une liberté sexuelle chez les Rwa, au contraire des mœurs des Maasaï des plaines environnantes.
} 
sexuelles, pendant cette période, avec ses autres femmes. Mais la fin de la polygamie mit fin à cet exutoire, et la période d'abstinence sexuelle postnatale fut raccourcie, d'autant que les conceptions de l'Eglise en matière de vie familiale encourageaient le rapprochement et la multiplicité des naissances chez les familles chrétiennes. Ces nouveaux comportements, conjugués à l'amélioration globale de la situation économique, ont sans doute contribué à l'accroissement démographique des $\mathrm{Rwa}^{15}$, qui aboutit à la situation foncière préoccupante qu'ils connaissent aujourd'hui.

\section{Les comportements alimentaires}

La densification démographique, sur le Mont Meru, eut des conséquences directes sur l'élevage, qui entraînèrent à leur tour une modification des comportements alimentaires. Les pâturages collectifs disparurent peu à peu pour faire place aux cultures, et c'est un élevage en stabulation qui prit le relais ${ }^{16}$. Après l'indépendance, les races de vaches anciennes furent croisées ou abandonnées au profit de vaches frisonnes, plus exigeantes en nourriture mais aussi bien meilleures laitières. L'objectif n'était plus d'avoir un troupeau important pour assurer une grosse consommation de viande et d'importants échanges d'animaux, mais de se limiter à quelques bonnes laitières dans une optique commerciale.

En effet la demande de lait n'a cessé de croître dans la ville proche d'Arusha, dont la population augmente constamment. Cette demande est d'autant plus forte que, sous l'influence britannique, le goût pour le thé au lait sucré est devenu général en Tanzanie. C'est aujourd'hui la boisson la plus courante dans les familles, et celle qu'on offre à l'arrivée d'un hôte. Les Rwa sont donc devenus producteurs de lait pour satisfaire cette demande citadine. Ils sont aujourd'hui, comme les autres Tanzaniens, buveurs de thé au lait alors qu'ils ne cultivent pas le thé, et par contre ils ne boivent pas, sauf exception, le café qu'ils produisent à vaste échelle. Ce paradoxe est un autre exemple d'arbitraire en matière alimentaire.

L'Eglise, bien sûr, encourage la consommation de thé, car ce n'est pas une boisson alcoolique. Lorsque des paroissiens se réunissent, c'est du thé que l'on prépare. L'influence morale de l'Eglise est devenue telle aujourd'hui que la consommation de bière de banane perd de plus en plus de terrain dans les réunions familiales ou publiques. Lors des mariages, elle est de plus en plus souvent remplacée par de multiples caisses de soda en bouteille, fabriqué sous licence américaine. On boit surtout du pepsi ou du mirinda de la Pepsi Cola Company, et ces sodas sont aussi offerts dans les

\footnotetext{
${ }_{16}^{15}$ Leur population est passée de 11666 en 1921 à 76375 en 1967, selon Spear (1997:128).

${ }^{16}$ Spear (1997:135).
} 
occasions où l'on donnait auparavant de la bière, telles que les demandes, annonces et paiements de mariage. Les réunions religieuses, aujourd'hui nombreuses, ne peuvent être accompagnées que de thé au lait ou de soda. Et mêmes les cérémonies générationnelles ${ }^{17}$, qui sont normalement l'occasion de grandes beuveries, ne sont organisées, dans certains cas où la plupart des intéressés sont des luthériens convaincus, qu'à la condition que seules des boissons non alcoolisées soient servies.

\section{CONCLUSION}

La bière a donc largement perdu son rôle de boisson sociale, qu'on offre pour formuler une demande de mariage ou autre, rassembler les anciens pour débattre d'un problème familial ou politique. Son rôle social s'est réduit, nombre de Rwa ont renoncé à en boire, mais les buveurs de bière n'ont pas pour autant disparu.

Ils restent nombreux à se réunir, surtout en fin d'après-midi, dans des débits de boisson spécialisés, où la bière est fabriquée, vendue et bue chaque jour en quantités énormes, comme chez les Chaga ${ }^{18}$. Les clients, dont beaucoup sont très assidus, sont surtout des hommes, auxquels s'adjoignent quelques rares femmes. Même si la bière de banane est bon marché, des sommes importantes y sont englouties. La bière est servie dans de grands pots de matière plastique et l'ébriété des consommateurs, en fin de journée, donne souvent lieu à des altercations aux abords de ces bars populaires. Leur clientèle n'a rien à voir avec celle, plus fortunée, d'une autre catégorie moins nombreuse de débits de boisson, ceux où l'on vend uniquement de la bière en bouteille, qui est beaucoup plus chère.

La consommation d'autres types d'alcool s'est aussi répandue, notamment parmi les jeunes gens. Les hommes âgés déplorent ces nouvelles pratiques qui n'ont d'autre but que l'ivresse, alors qu'auparavant seuls les hommes d'âge mûr buvaient, pour le plaisir d'être ensemble et non pour se saoûler. Ces nouveaux alcools sont un alcool à base de mélasse, le pure (terme emprunté à l'anglais), appelé gongo en swahili, et l'alcool de maïs des Masaai et des Arusha, le dadii.

En même temps que ces nouvelles manières de boire, les vieux déplorent ce qu'ils perçoivent comme une dégradation générale des mœurs : la perte du respect pour les aînés, la diffusion de la délinquance, la baisse générale de moralité qui se traduit en particulier dans le comportement des jeunes filles. Beaucoup d'entre elles deviennent mères avant le mariage, ce

17 Elles ont connu un regain de vitalité à la faveur d'un conflit religieux récent au sein de l'Eglise luthérienne (Baroin 1996).

18 Moore (1986:351). 
qui eut été impensable une génération plus tôt. Cette évolution, de façon générale, est mal ressentie, et ces grossesses sont très mal perçues, d'autant que l'Eglise ne manque pas de condamner, elle aussi, une telle inconduite. Mais comme ces jeunes femmes, rejetées par leur famille, n'ont pas d'autre ressource que de se prostituer en ville pour survivre, l'Eglise luthérienne intercède maintenant en leur faveur auprès de leurs parents pour convaincre ceux-ci de ne plus les chasser, ne serait-ce que pour sauver les âmes des enfants qu'elles mettent au monde.

Ce que l'Eglise, et avec elle une bonne partie de la population, perçoit comme une perte de moralité et un dérèglement général des comportements apparaît clairement dans l'évolution des manières de boire. Ils sont le fruit d'un bouleversement trop rapide des structures sociales, auquel les mentalités ont du mal à s'adapter. Ils sont aussi liés, pour beaucoup de familles, à un manque de perspectives économiques, en raison de l'explosion démographique et d'une pression foncière devenue excessive sur les flancs du Mont Meru, alors que les formations professionnelles qualifiantes débouchant sur des emplois nouveaux manquent cruellement pour la plupart des jeunes. Tel est le terreau sur lequel se nourrit aussi bien l'alcoolisme des uns, que l'intégrisme (chrétien) polémique des autres.

\section{Références bibliographiques}

BAROIN, Catherine, 1996, Religious conflict in 1990-1993 among the Rwa : secession in a Lutheran diocese in Northern Tanzania, African Affairs, 95, n 381, pp.529-554.

-, 1998, Introduction et culture du café chez les Rwa de Tanzanie du Nord, in M. Chastanet (éd.), Plantes et paysages d'Afrique. Une histoire à explorer. Paris, Karthala et Orstom, pp.529-549.

-, (sous presse), Eleusine et maïs, cent ans de cuisine des céréales chez les Rwa (Tanzanie du Nord), in M. Chastanet, D. Juhé-Beaulaton et F.-X. Fauvelle-Aymar (éds.), Cuisine et société en Afrique : histoire, saveurs, savoir-faire, Paris, Karthala.

CORY, Hans, non daté, Adanyaki Emoti (Breaking the cooking pot), Cory Paper $\mathrm{n}^{\circ} 33,3$ pages dactyl.

HARJULA, Raimo, 1969, God and the sun in Meru thought, Annals of the Finnish society for missiology and ecumenics, Helsinki.

-, 1978, Ancestral spirits as helpers among the Meru of Tanzania, Temenos (Helsinki, Finlande), 14, pp.53-78.

,- 1989 , Curse as a manifestation of broken human relationships among the Meru of Tanzania, in A. Jacobson-Widding et D. Westerlund, (éds.), Culture, experience and pluralism. Essays on african ideas of 
illness and healing. Uppsala: Acta Universitatis Upsaliensis, Uppsala Studies in cultural anthropology 13, pp.125-137.

-, 1997, The ideal of the good life, as crystallized by Tanzanian Meru proverbs, Studia orientalia, Helsinki, 81.

ILIFFE, John, 1979, A Modern History of Tanganyika, Cambridge : Cambridge University Press.

JAPHET, Kirilo et SEATON, Earl, 1967, The Meru Land Case, Nairobi, East african publishing house.

JOLLY, Eric, 1995, La bière de mil dans la société dogon, thèse de doctorat d'ethnologie, Université de Paris X-Nanterre, 2 vol.

KAAYA, Baltazar, 1978, The planting of christianity in Meru. Its conflicts and similarities with the traditional culture of the Wameru, Mémoire du Makumira Lutheran Theological College.

MOORE, Sally Falk, 1986, Social facts and fabrications. "Customary" law on Kilimanjaro, 1880-1980, Cambridge, Cambridge University Press.

PEATRIK, Anne-Marie (éd.), 1995, Ages et générations: ordres et désordres. Des sociétés au rythme du temps, L'homme, 134.

-, 1999, La vie à pas contés. Génération, âge et société dans les hautes terres du Kénya (Meru Tigania-Igembe), Nanterre, Société d'ethnologie.

PURITT, Paul, 1970, The Meru of Tanzania : a study of their social and political organization, Ph. D., University of Illinois at Urbana Champaign.

SPEAR, Thomas (éd.), 1995, Evangelisch-Lutherisches Missionsblatt. Extracts on Arusha and Meru, 1897-1914, African Studies Program, University of Wisconsin-Madison.

-, 1997, Mountain Farmers. Moral Economies of Land and Agricultural Development in Arusha and Meru, Oxford : James Currey / Dar-es-Salaam : Mkuki na Nyota / Berkeley, University of California Press.

Tanganyika Territory, Annual reports of the provincial commissioners, 1929-1959.

Tanganyika, 1954, "Local government Memoranda", $\mathrm{n}^{\circ}$ 1, part 1, Dares-Salaam, Government Printer. 